\title{
Analysis of risk factors for portal vein thrombosis after liver resection
}

\author{
Jae Hyun Han, Dong-Sik Kim, Young Dong Yu, Sung Won Jung, Young In Yoon, Hye Sung Jo \\ Division of HBP Surgery and Liver Transplantation, Department of Surgery, Korea University College of Medicine, Seoul, Korea
}

\begin{abstract}
Purpose: We evaluated the risk factors for posthepatectomy thrombosis including portal vein thrombosis (PVT) and clinical outcomes.

Methods: We retrospectively analyzed 563 patients who had undergone hepatectomy from February 2009 to December 2014. Twenty-nine patients with preoperatively confirmed thrombosis and tumor recurrence-related thrombosis were excluded. We identified the location of the thrombosis as main portal vein (MPV), peripheral portal vein (PPV) and other site such as hepatic vein or inferior vena cava. Patients with MPV thrombosis and PPV thrombosis with main portal flow disturbance were treated with anticoagulation therapy. We performed operative thrombectomy before anticoagulation therapy who did combined portal vein (PV) segmental resection.

Results: Of the 534 patients, 22 (4.1\%) developed posthepatectomy thrombosis after hepatectomy. Among them, 19 (86.4\%) had PVT. The mean duration of Pringle's maneuver was significant longer in the PVT group than the no-thrombosis group $(P=0.020)$. Patients who underwent combined $P V$ segmental resection during hepatectomy were more likely to develop posthepatectomy PVT $(P=0.001)$. Thirteen patients who had MPV thrombosis and PPV thrombosis with main portal flow disturbance received anticoagulation therapy immediately after diagnosis and all of them were improved. Among them, 2 patients who developed PVT at the PV anastomosis site after PV segmental resection, underwent operative thrombectomy before anticoagulation therapy and both were improved. There were no patients who developed complications related to anticoagulation therapy.

Conclusion: Long duration of Pringle's maneuver and PV segmental resection were risk factors. Anticoagulation therapy or operative thrombectomy should be considered for PVT without contraindications.

[Ann Surg Treat Res 2019;96(5):230-236]
\end{abstract}

Key Words: Hepatectomy, Portal vein, Thrombosis, Risk factors, Treatment outcome

\section{INTRODUCTION}

Hepatectomy remains the standard treatment option for benign and malignant primary liver disease as well as metastatic diseases such as metastasis from colorectal cancer [1,2]. Advances in operative techniques and perioperative management have reduced the morbidity and mortality rate amongst hepatectomy patients; however, the postoperative complication rate is still relatively high [3,4]. Recent studies reported an overall complication rate ranging from $20 \%$ to over $40 \%$ and major complications arise in $10 \%$ to $20 \%$ of all cases with complications $[5,6]$. Posthepatectomy liver failure, bile leakage, pulmonary complications, deep vein thrombosis, and portal vein thrombosis (PVT) are considered major complications that could lead to post-operative death [7-11].

Posthepatectomy PVT is a potentially life-threatening compli-
Received November 16, 2018, Revised January 11, 2019,

Accepted January 29, 2019

\section{Corresponding Author: Dong-Sik Kim}

Division of HBP Surgery and Liver Transplantation, Department of Surgery, Korea University College of Medicine, 73 Inchon-ro, Seongbuk-gu, Seoul 02841, Korea

Tel: +82-2-920-6620, Fax: +82-2-921-6620

E-mail: kimds1@korea.ac.kr

ORCID code: https://orcid.org/0000-0002-0608-1580
Copyright (c) 2019, the Korean Surgical Society

(C) Annals of Surgical Treatment and Research is an Open Access Journal. All articles are distributed under the terms of the Creative Commons Attribution NonCommercial License (http://creativecommons.org/licenses/by-nc/4.0/) which permits unrestricted non-commercial use, distribution, and reproduction in any medium, provided the original work is properly cited. 
cation that can reduce portal flow to the liver and lead to the development of mesenteric ischemia when it extends to the superior mesenteric vein. Its clinical importance has been described in some liver transplantation literatures $[12,13]$, but there have only been a few studies regarding hepatectomy $[11,14]$. For these reasons, it has been often overlooked and treatment options have not yet been established.

The aim of the present study was to evaluate the incidence, risk factors, treatment options, and clinical outcomes of posthepatectomy thrombosis, especially PVT.

\section{METHODS}

We retrospectively reviewed the medical records of 563 patients who had undergone hepatic resection for various diseases from February 2009 to December 2014 at our hospital. Posthepatectomy thrombosis was defined as thrombosis that occurred in the portal vein, hepatic vein, or inferior vena cava (IVC) as seen on postoperative imaging studies. We excluded 29 patients with thrombosis confirmed preoperatively on imaging studies and tumor recurrence-related thrombosis from the posthepatectomy thrombosis group.

This study was approved by the Institutional Review Board of Korea University Anam Hospital (approval number: ED16061). Written informed consent was waived by the IRB.

\section{Surgical procedure}

Patients with liver cirrhosis corresponding to Child-Pugh classification $C$ were excluded from liver resection. In the indocyanine green (ICG) clearance test, we set the safe limit for the ICG retention value as $<15 \%$ at 15 minutes for major hepatectomy. For patients with an ICG retention value of $>15 \%$, we performed a partial hepatectomy [15].

We used the extrahepatic Glissonian approach in almost all cases when we performed resection greater than sectionectomy, however, in cases of hilar cholangiocarcinoma, we performed individual ligation because of lymph node dissection around the hepaticoduodenal ligament. Hepatic parenchymal resection was done using an ultrasonic dissector (CUSA; Integra Lifesiences, Plainsboro, NJ, USA) and the clamp-crushing technique as appropriate. For laparoscopic hepatectomy, we used laparoscopic CUSA and an ultrasonic scalpel (Harmonic ACE; Ethicon Endo-Surgery, Cincinnati, OH, USA). Small vessels were sealed with electrocautery, while large vessels and bile ducts, were ligated with a metal clip and Hem-o-lok clips (Weck, Research Triangle Park, NC, USA), and Glissonian pedicles were suture ligated.

We used Pringle's maneuver occasionally to reduce bleeding during hepatic parenchymal resection except in cases of cholangiocarcinoma. We exposed the Glisson's capsule and occluded it to be resected using a Rummel tourniquet. It was then subjected to 15 minutes of inflow occlusion followed by 5 minutes of reperfusion, repeated as necessary.

\section{Postoperative follow-up}

All patients were managed with a standardized treatment protocol. Contrast-enhanced abdominal CT was performed if patients showed abnormal liver function in the postoperative course or developed a high fever without any specific febrile focus. However, in the case of combined PV segmental resection, color Doppler ultrasonography was routinely performed the day after surgery to confirm the portal flow. We identified the location of the posthepatectomy thrombosis as follows: main portal vein (MPV) thrombosis was defined as PV thrombosis involving the MPV, peripheral portal vein (PPV) thrombosis was defined as thrombosis in the portal vein stump or a branch of the portal vein, and thrombosis in the hepatic vein or IVC was defined as an "other" group.

\section{Anticoagulation therapy}

Patients with posthepatectomy thrombosis were not given anticoagulation therapy routinely because hepatectomy can result in coagulopathy and increased bleeding. Almost all patients with MPV thrombosis were given anticoagulation therapy if there were no contraindications. In patients with PPV thrombosis, anticoagulation therapy was started when the thrombus at the portal vein stump extended to the MPV and when the portal vein branch thrombus developed portal flow disturbance or abnormal liver function. The patients who developed thrombosis at the PV anastomosis site after PV segmental resection, operative thrombectomy was performed and followed by anticoagulation therapy.

Anticoagulation therapy was accomplished with low molecular weight heparin followed by oral warfarin in all treated patients. The target prothrombin time international normalized ratio was between 2 and 3. A contrast-enhanced CT scan was done monthly after treatment and anticoagulation therapy was continued until the thrombosis was resolved on a follow-up imaging study.

\section{Statistical analysis}

Mean, standard deviation, and ranges were used to present numerical variables. Continuous variables were compared by Student t-test. Differences in categorical variables were analyzed with the chi-square test. The Cox regression hazard ratio model analysis was used for identification of independent risk factors. P-values of $<0.05$ were considered to indicate statistical significance. 


\section{RESULTS}

\section{Patient characteristics}

Of the 534 patients, 22 (4.1\%) developed thrombosis either in the portal vein, hepatic vein, or IVC after hepatectomy. The most common site where thrombosis developed was the portal vein ( $\mathrm{n}=19,3.6 \%$ of total). The mean age of all patients was $57.4 \pm 13.1$ years (18-95 years). Three hundred fifty-seven patients $(66.9 \%)$ were male. The most common underlying disease was hepatocellular carcinoma (39.9\%), followed by metastatic tumor from colorectal cancer (22.8\%). One hundred ninety-two patients (36.0\%) required resection more than 2 liver sections. The mean operative time was $317.5 \pm 161.3$ minutes. The number of patients who underwent Pringle's maneuver was 271 (50.7\%), and the mean duration of Pringle's maneuver was $17.9 \pm 21.7$ minutes (Table 1).

\section{Comparative results between the PVT and no- thrombosis groups}

We analyzed the risk factors for posthepatectomy PVT, which was the most common site of posthepatectomy thrombosis in this study.

The mean duration of Pringle's maneuver was significant longer in the posthepatectomy PVT group $(\operatorname{Exp}(B), 1.205$; 95\% confidence interval [CI], 1.029-1.411; $\mathrm{P}=0.020$ ). The hepatectomy accompanied with portal vein resection was also significantly influenced the development of posthepatectomy PVT (Exp(B), 9.361; 95\% CI, 2.550-34.368; P = 0.001).

More patients in the posthepatectomy PVT group had resec- ted of a large volume of the liver $(52.6 \%$ vs. $35.2 \%)$, but the difference was not statistically significant $(P=0.311)$. The mean duration of operative time tended to be longer, in the posthepatectomy PVT group, but this difference was also not statistically significant $(\mathrm{P}=0.752)$ (Table 2$)$.

Table 1. Patient characteristics $(n=534)$

\begin{tabular}{lc}
\hline \multicolumn{1}{c}{ Characteristic } & Value \\
\hline Age $(y r)$ & $57.4 \pm 13.1$ \\
Male sex & $357(66.9)$ \\
Disease & \\
Hepatocellular carcinoma & $213(39.9)$ \\
Cholangiocarcinoma & $63(11.8)$ \\
Colorectal cancer metastasis & $122(22.8)$ \\
Benign diseases & $136(25.5)$ \\
Location of thrombosis & $22(4.1)$ \\
Portal vein & $19(3.6)$ \\
Others & $3(0.5)$ \\
Laparoscopic surgery & $66(12.4)$ \\
Large resection & $192(36.0)$ \\
Operation time (min) & $317.5 \pm 161.3$ \\
Pringle's maneuver & $271(50.7)$ \\
Number & $1.19 \pm 1.44$ \\
Time (min) & $17.9 \pm 21.7$ \\
Portal vein resection & $18(3.4)$ \\
\hline
\end{tabular}

Values are presented as mean \pm standard deviation or number (\%).

HCC, hepatocellular carcinoma.

${ }^{a)}$ Resection more than 2 liver sections.

Table 2. Risk factor analysis of portal vein (PV) thrombosis group

\begin{tabular}{|c|c|c|c|c|}
\hline Variable & No thrombosis & PV thrombosis & P-value & Odd ratio \\
\hline No. of patients & $512(95.9)$ & $19(3.6)$ & & \\
\hline Age $(y r)$ & $57.3 \pm 13.2$ & $59.1 \pm 11.7$ & 0.560 & \\
\hline Male sex & $342(66.8)$ & $13(68.4)$ & 0.883 & \\
\hline \multicolumn{5}{|l|}{ Disease } \\
\hline $\mathrm{HCC}$ & $201(39.3)$ & $12(63.2)$ & 0.110 & \\
\hline Cholangiocarcinoma & $57(11.1)$ & $5(26.3)$ & 0.355 & \\
\hline Colon cancer with liver metastasis & $120(23.4)$ & $0(0)$ & 0.996 & \\
\hline Others & $134(26.2)$ & $2(10.5)$ & 0.144 & \\
\hline Laparoscopic surgery & $65(12.7)$ & $1(5.3)$ & 0.353 & \\
\hline Large resection $^{\text {a) }}$ & $180(35.2)$ & $10(52.6)$ & 0.311 & \\
\hline Operation time (min) & $314.9 \pm 157.3$ & $359.2 \pm 234.5$ & 0.752 & \\
\hline \multicolumn{5}{|l|}{ Complications } \\
\hline Bile leakage & $5(1.0)$ & $1(5.3)$ & 0.123 & \\
\hline Pringle's maneuver & $259(50.6)$ & $12(63.2)$ & 0.764 & \\
\hline Number & $1.17 \pm 1.43$ & $1.32 \pm 1.46$ & 0.662 & \\
\hline Time (min) & $17.7 \pm 21.4$ & $21.1 \pm 24.4$ & 0.020 & $1.205(1.029-1.411)$ \\
\hline Portal vein resection & $14(2.7)$ & $4(21.1)$ & 0.001 & $9.361(2.550-34.368)$ \\
\hline
\end{tabular}

Values are presented as number (\%) or mean \pm standard deviation.

HCC, hepatocellular carcinoma.

${ }^{\text {a) }}$ Resection more than 2 liver sections. 


\section{Clinical outcomes}

Of the 22 patients who developed posthepatectomy thrombosis, 13 (59.1\%) received anticoagulation therapy. Twelve of the 13 patients clinically improved, and, of 9 patients who did not receive anticoagulation therapy, 8 patients improved. There were no patients who developed complications related to anticoagulation therapy during a mean treatment period of $12.5 \pm 2.9$ weeks (median, 12 weeks). There was no mortality related to posthepatectomy thrombosis and no cases of thrombosis progression.

We analyzed the patients by the site of posthepatectomy thrombosis. Of the 6 patients who developed thrombosis in the MPV, 5 were treated with anticoagulation therapy and all cases were resolved (Fig. 1). Of the 5 patients who were treated with anticoagulation therapy, 2 underwent PV segmental resection because of tumor invasion and developed PVT 1 day after the operation. PVT was detected on routine postoperative Doppler sonography at the anastomosis site and the patients underwent operative thrombectomy followed by anticoagulation therapy. Another 2 patients who underwent PV segmental resection, PVT developed in the PPV and treated only with anticoagulation therapy. In the one who did not receive anticoagulation therapy in the MPV group, thrombosis developed at the junction of the right and left portal vein, adjacent to the resection margin, 7 days after right posterior sectionectomy. Because of the appearance of coagulopathy after hepatectomy, and because it did not affect portal flow to the remaining parts of the liver, the patient did not receive anticoagulation therapy and PVT spontaneously resolved 1 month after hepatectomy.

Of the 13 patients who developed thrombosis in the PPV, 6 were treated with anticoagulation therapy and all cases were

Table 3. Clinical outcomes

\begin{tabular}{cc}
\hline Anticoagulation therapy & $\begin{array}{c}\text { Clinical outcome, } n(\%) \\
\text { (improved) }\end{array}$ \\
\hline Total $(n=22)$ & $21(95.5)$ \\
Yes $(n=13,59.1 \%)$ & $13(100)$ \\
No $(n=9,40.9 \%)$ & $8(88.9)$ \\
MPV $(n=6,27.3 \%)$ & $6(100)$ \\
Yes $\left(n=5^{\text {a) }}, 83.3 \%\right)$ & $5(100)$ \\
No $(n=1,16.7 \%)$ & $1(100)$ \\
PPV $(n=13,59.1 \%)$ & $12(92.3)$ \\
Yes $(n=6,46.2 \%)$ & $6(100)$ \\
No $(n=7,53.8 \%)$ & $6(85.7)$ \\
Others $(n=3,13.6 \%)$ & $3(100)$ \\
Yes $(n=2,66.7 \%)$ & $2(100)$ \\
No $(n=1,33.3 \%)$ & $1(100)$ \\
\hline
\end{tabular}

MPV, main portal vein; PPV, peripheral portal vein.

${ }^{a)}$ Two patients underwent thrombectomy followed by anticoagulation therapy.
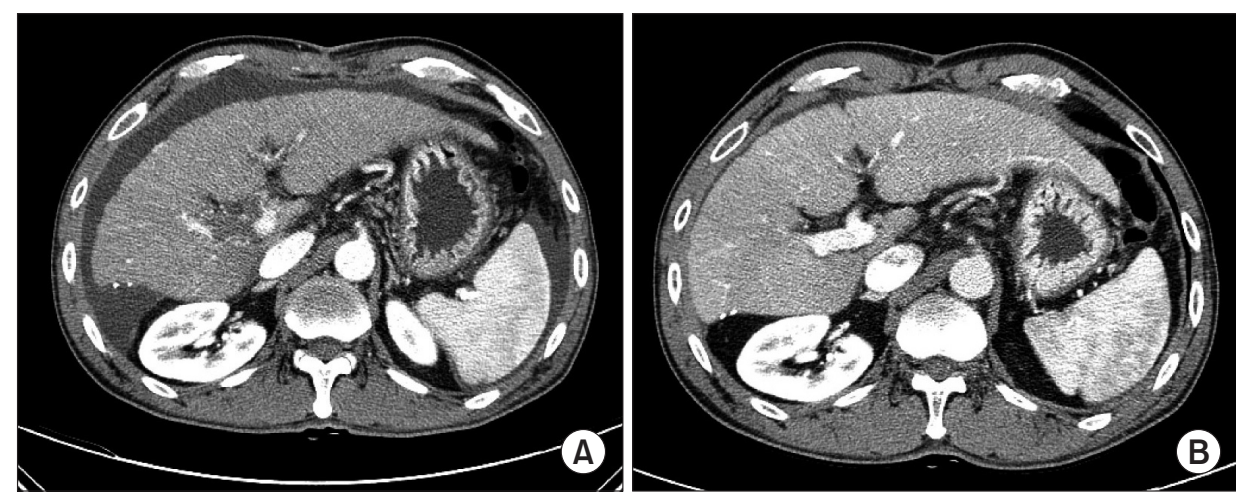

Fig. 1. (A) Main portal vein thrombosis was discovered on the third day after hepatectomy and (B) it was disappeared after 8 months of anticoagulation therapy.
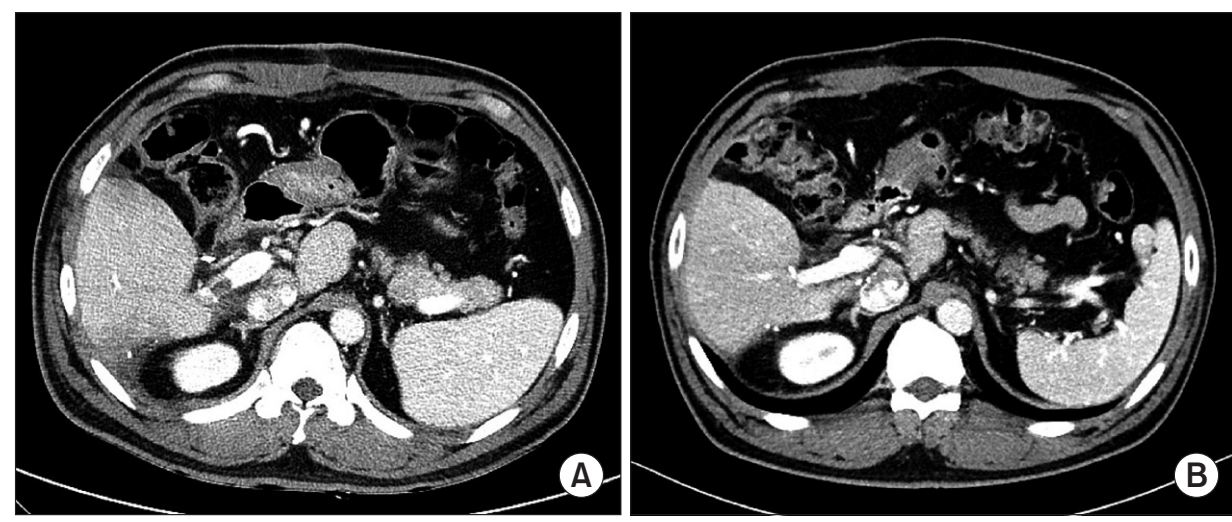

Fig. 2. (A) Peripheral portal vein thrombosis was discovered on the first week after hepatectomy and (B) it was disappeared after 3 months of anticoagulation therapy. 
resolved (Fig. 2). Of the 7 patients who did not receive anticoagulation therapy, 6 improved. In the 7 patients who did not receive anticoagulation therapy, thrombosis developed at the portal vein stump or peripheral branch of the portal vein as evidenced on a routine follow-up abdominal CT done 7 days after the operation, and there was no disturbance of portal flow to the liver or liver function during the postoperative course. In all patients, thrombosis spontaneously resolved on the followup imaging study without anticoagulation therapy.

Of the 3 patients who developed thrombosis at other sites after hepatectomy, 2 developed thrombosis in the IVC after IVC replacement due to tumor invasion and the other developed thrombosis of the peripheral hepatic vein adjacent to the resection margin. The 2 patients who developed thrombosis in the IVC improved after anticoagulation therapy and the other patient, who developed thrombosis in the peripheral hepatic vein, improved without treatment for a similar reason as the no-treatment group of peripheral PVT patients (Table 3).

\section{DISCUSSION}

PVT is known to be a common complication of liver cirrhosis $[16,17]$ and has been reported in several studies after operations such as liver transplantation and hepatectomy [11-14]. It is considered a potentially life-threatening complication that can reduce portal flow, decrease liver function, and aggravate portal hypertension. It may also lead to mesenteric ischemia and sepsis when it extends to the superior mesenteric vein [18].

In this study, $4.1 \%$ of the patients developed posthepatectomy thrombosis and, among them, $86.4 \%$ developed thrombosis in the portal vein (3.6\% of the total number of patients). Previous studies of liver transplant patients reported the incidence of PVT as $5 \%-16 \%$, and that of hepatectomy as $2 \%-9 \%$ [11-14]. Although the incidence of PVT after hepatectomy is lower than that of other complications, such as liver failure (4\%-19 \%) $[19,20]$, pulmonary complications (13\%) [9], and venous thromboembolism (2.9\%) [10], it is not rare.

Yoshiya et al. [11] recently reported that Pringle's maneuver can result in portal vein endothelial injury and stasis, so the duration of Pringle's maneuver could be a significant risk factor for PVT. In this study, the mean duration of Pringle's maneuver was also the significant risk factor related to the operation for post-hepatectomy PVT.

Twenty-eight patients underwent combined portal vein segmental resection during hepatectomy because of tumor invasion. Among them, 4 patients developed posthepatectomy PVT (22.2\%) and it is significantly higher than no thrombosis group $(P=0.001)$. This result is supported by previous studies on PVT after pancreatic resection with portal vein reconstruction, which reported that the rate of PVT is $13 \%-17 \%$ $[21,22]$, which is significantly higher than that reported by other studies without portal vein reconstruction [23]. The etiology of PVT after portal vein segmental resection was found to be similar in several studies on PVT after splenectomy [24]. Surgically induced endothelial damage or venous stasis accompanied by liver cirrhosis or portal hypertension could lead to PVT.

Some investigators recently reported that large resection volume, especially right hepatectomy, is an independent risk factor associated with PVT [11,14]. This is supported by the etiology of PVT based on one of Virchow's triad (venous stasis, hypercoagulability, and endothelial injury), venous stasis. The cause of PVT in liver cirrhosis patients may involve venous stasis induced by portal hypertension after hepatectomy with large resection volume [25]. The present study revealed that more patients in the posthepatectomy PVT group underwent resection of more than 2 liver sections (35.2\% vs. 52.6\%), but the difference was not statistically significant $(\mathrm{P}=0.119)$.

We mainly used color Doppler ultrasonography and contrastenhanced CT scans for PVT diagnosis and follow-up. We choose a diagnostic modality based on level of suspicion. If it was more likely to develop PVT such as combined PV segmental resection or long duration of Pringle's maneuver with abnormal liver function, Doppler ultrasonography was preferred. Several previous studies also reported the usefulness of color Doppler ultrasonography because of high sensitivity $(93 \%)$ and specificity $(99 \%)[26,27]$. However, there are some reports that the sensitivity and specificity of contrast-enhanced CT are not inferior to those of Doppler ultrasonography for detection of PVT [14] and in the postoperative setting it is difficult to detect PVT because of the upper abdominal incision, adhesion around the liver hilum, and increased amount of gas in the bowel. Therefore, when the patients showed some problems after hepatectomy not specific to PVT, contrast-enhanced CT would be useful and not inferior to Doppler ultrasonography to detect PVT.

There have been a few studies on the natural course of extrahepatic nonmalignant PVT after hepatectomy. Luca et al. [28] reported the natural course of PVT in patients with liver cirrhosis but not malignancy who did not receive anticoagulation therapy. They reported that PVT improved in $45 \%$ of patients without treatment. In a recent study, $60 \%$ of patients improved without treatment [11]. However, many studies have recommended that anticoagulation therapy should be begun immediately after a diagnosis of PVT $[13,18,29]$. In this study, 8 of 22 patients $(36.3 \%)$ improved without anticoagulation therapy; however, their condition was because of coagulopathy following hepatectomy and did not affect portal flow to the remaining parts of the liver.

We employed anticoagulation therapy consisting of low molecular weight heparin followed by oral warfarin, as many other studies have described $[13,18,29]$. Actually, there is a 
controversy regarding the complete recanalization rate of anticoagulation therapy, which ranges from $24 \%$ to $81 \%$ [30], and some reports recommended more invasive therapy such as operative thrombectomy [14]. In the present study, almost all patients who developed PVT did so in the MPV, and 6 of 13 patients who developed PVT in the PPV received anticoagulation therapy immediately after the diagnosis of PVT because of the possibility of decreased portal flow. Two of the patients who developed PVT in the MPV after combined PV segmental resection underwent operative thrombectomy and followed by anticoagulation therapy. All treated patients recovered during the follow-up period without any complications related to thrombectomy or anticoagulation therapy.

In conclusion, PVT after hepatectomy is not rare and is a potentially life-threatening complication that need careful attention. In this study, the long duration of Pringle's maneuver and combined PV segmental resection was a significant risk factors for posthepatectomy PVT.
Anticoagulation therapy is a safe treatment option and is recommended immediately after the diagnosis of PVT for all patients without contraindications, especially for MPV thrombosis and PPV thrombosis with main portal flow disturbance. However, operative thrombectomy should be conducted before anticoagulation therapy for the PVT after PV segmental resection which potentially cause total portal flow obliteration.

\section{CONFLICTS OF INTEREST}

No potential conflict of interest relevant to this article was reported.

\section{ACKNOWLEDGEMENTS}

Supported by the National Research Foundation of Korea (NRF), funded by the Ministry of Education, Science, and Technology (2014R1A1A1A05006371).

\section{REFERENCES}

1. Poon RT, Fan ST, Lo CM, Liu CL, Lam CM, Yuen WK, et al. Improving perioperative outcome expands the role of hepatectomy in management of benign and malignant hepatobiliary diseases: analysis of 1222 consecutive patients from a prospective database. Ann Surg 2004:240:698-708.

2. Xu J, Wei Y, Zhong Y, Fan J, Zhou J, Qin L, et al. Hepatectomy for liver metastasis of colorectal cancer. Int J Colorectal Dis 2009:24:419-25.

3. Asiyanbola B, Chang D, Gleisner AL, Nathan H, Choti MA, Schulick RD, et al. Operative mortality after hepatic resection: are literature-based rates broadly applicable? J Gastrointest Surg 2008;12:84251.

4. Jarnagin WR, Gonen M, Fong Y, DeMatteo RP, Ben-Porat L, Little S, et al. Improvement in perioperative outcome after hepatic resection: analysis of 1,803 consecutive cases over the past decade. Ann Surg 2002;236:397-406.

5. Benzoni E, Cojutti A, Lorenzin D, Adani GL, Baccarani U, Favero A, et al. Liver resective surgery: a multivariate analysis of postoperative outcome and complication.
Langenbecks Arch Surg 2007:392:45-54.

6. Sadamori H, Yagi T, Shinoura S, Umeda Y, Yoshida R, Satoh D, et al. Risk factors for major morbidity after liver resection for hepatocellular carcinoma. Br J Surg 2013; 100:122-9.

7. Rahbari NN, Garden OJ, Padbury R, Brooke-Smith M, Crawford M, Adam R, et al. Posthepatectomy liver failure: a definition and grading by the International Study Group of Liver Surgery (ISGLS). Surgery 2011;149:713-24.

8. Koch M, Garden OJ, Padbury R, Rahbari NN, Adam R, Capussotti L, et al. Bile leakage after hepatobiliary and pancreatic surgery: a definition and grading of severity by the International Study Group of Liver Surgery. Surgery 2011;149:680-8.

9. Nobili C, Marzano E, Oussoultzoglou E, Rosso E, Addeo P, Bachellier P, et al. Multivariate analysis of risk factors for pulmonary complications after hepatic resection. Ann Surg 2012;255:540-50.

10. Turley RS, Reddy SK, Shortell CK, Clary BM, Scarborough JE. Venous thromboembolism after hepatic resection: analysis of 5,706 patients. J Gastrointest Surg 2012;
16:1705-14

11. Yoshiya S, Shirabe K, Nakagawara H, Soejima Y, Yoshizumi T, Ikegami T, et al. Portal vein thrombosis after hepatectomy. World J Surg 2014;38:1491-7.

12. Wozney P, Zajko AB, Bron KM, Point S, Starzl TE. Vascular complications after liver transplantation: a 5-year experience. AJR Am J Roentgenol 1986;147:657-63.

13. Woo DH, Laberge JM, Gordon RL, Wilson MW, Kerlan RK Jr. Management of portal venous complications after liver transplantation. Tech Vasc Interv Radiol 2007; 10:233-9.

14. Kuboki S, Shimizu H, Ohtsuka M, Kato A, Yoshitomi H, Furukawa K, et al. Incidence, risk factors, and management options for portal vein thrombosis after hepatectomy: a 14-year, single-center experience. Am J Surg 2015;210:878-85.e2.

15. Lam CM, Fan ST, Lo CM, Wong J. Major hepatectomy for hepatocellular carcinoma in patients with an unsatisfactory indocyanine green clearance test. Br J Surg 1999:86:1012-7.

16. Kinjo N, Kawanaka H, Akahoshi T, Matsumoto Y, Kamori M, Nagao Y, et al. Portal 
vein thrombosis in liver cirrhosis. World J Hepatol 2014;6:64-71.

17. Okuda K, Ohnishi K, Kimura K, Matsutani S, Sumida M, Goto N, et al. Incidence of portal vein thrombosis in liver cirrhosis. An angiographic study in 708 patients. Gastroenterology 1985;89:279-86.

18. Thomas RM, Ahmad SA. Management of acute post-operative portal venous thrombosis. J Gastrointest Surg 2010;14:570-7.

19. Capussotti L, Muratore A, Amisano M, Polastri R, Bouzari H, Massucco P. Liver resection for hepatocellular carcinoma on cirrhosis: analysis of mortality, morbidity and survival--a European single center experience. Eur J Surg Oncol 2005:31:986-93.

20. Chok KS, Ng KK, Poon RT, Lo CM, Fan ST. Impact of postoperative complications on long-term outcome of curative resection for hepatocellular carcinoma. Br J Surg 2009;96:81-7.

21. Smoot RL, Christein JD, Farnell MB. Durability of portal venous reconstruction following resection during pancreaticoduo- denectomy. J Gastrointest Surg 2006;10: 1371-5.

22. Glebova NO, Hicks CW, Piazza KM, Abularrage CJ, Cameron AM, Schulick RD, et al. Technical risk factors for portal vein reconstruction thrombosis in pancreatic resection. J Vasc Surg 2015;62:424-33.

23. Ansari D, Ansorge C, Andren-Sandberg A, Ansari D, Segersvard R. Portal venous system thrombosis after pancreatic resection. World J Surg 2013;37:179-84.

24. Fujita F, Lyass S, Otsuka K, Giordano L, Rosenbaum DL, Khalili TM, et al. Portal vein thrombosis following splenectomy: identification of risk factors. Am Surg 2003;69:951-6.

25. Garcia-Pagan JC, Hernandez-Guerra M, Bosch J. Extrahepatic portal vein thrombosis. Semin Liver Dis 2008;28:282-92.

26. Tessler FN, Gehring BJ, Gomes AS, Perrella RR, Ragavendra N, Busuttil RW, et al. Diagnosis of portal vein thrombosis: value of color Doppler imaging. AJR Am J Roentgenol 1991;157:293-6.
27. Bach AM, Hann LE, Brown KT, Getrajdman GI, Herman SK, Fong Y, et al. Portal vein evaluation with US: comparison to angiography combined with CT arterial portography. Radiology 1996;201:149-54.

28. Luca A, Caruso S, Milazzo M, Marrone G, Mamone G, Crino F, et al. Natural course of extrahepatic nonmalignant partial portal vein thrombosis in patients with cirrhosis. Radiology 2012;265:124-32.

29. Condat B, Pessione F, Hillaire S, Denninger $\mathrm{MH}$, Guillin $\mathrm{MC}$, Poliquin $\mathrm{M}$, et al. Current outcome of portal vein thrombosis in adults: risk and benefit of anticoagulant therapy. Gastroenterology 2001; 120:490-7.

30. Condat B, Pessione F, Helene Denninger M, Hillaire S, Valla D. Recent portal or mesenteric venous thrombosis: increased recognition and frequent recanalization on anticoagulant therapy. Hepatology 2000;32:466-70. 